\title{
Nonlinear Microwave System Characterization Based on Higher Order Statistics
}

\author{
João Paulo Martins, Nuno Borges Carvalho and José Carlos Pedro \\ Instituto de Telecomunicações, Campo Universitário, 3810-193 Aveiro, Portugal \\ joaoptm@av.it.pt, nborges@det.ua.pt, jcpedro@det.ua.pt
}

\begin{abstract}
This paper presents a setup for the nonlinear vectorial characterization based on the computation of the input-output signals' Higher Order Statistics. This setup assures a high dynamic range in the measurement and a broad useful bandwidth. The phase reference presents no bandwidth constraints since it is obtained directly from the input signal. The proposed setup was validated by simulation and the results state the validity of the method.
\end{abstract}

\section{SUMMARY}

In the recent past it can be noticed an increasing interest in the nonlinear system characterization, especially in the RF and microwave area. This can be related to the increasing requirements in the design specifications, which demands an accurate representation of the RF and microwave systems not only in their linear outcome but especially the nonlinear response.

The nonlinear characterization is a challenging task since concepts such as phase and transfer function must be redefined. In [1-2] the phase concept is addressed and a relation between the phase of tones at different frequencies is given.

If the analysis is broaden, permitting now both linear and nonlinear behavior, the former linear transfer function concept is not valid. In this case, the output spectra is composed by the fundamental tones applied to the input and also by all the mixing products that can be obtained until a certain order, assumed as the nonlinear order of the system. Under this scenario, the transfer function is a mapping of a mixing combination of input tones into a certain output tone. This can be viewed as a $n$ order nonlinear transfer function, where the referred order is the one needed to obtain that spectral output from the considered input tones.

From the measurement point of view, the problem was addressed through several schemes [3-10]. In [3-7] a cancellation approach is adopted. The measurement procedure is complex since the cancellation requests a fine tuning both in amplitude and phase. Moreover, the extrapolation of the method to input signals with more than two tones is not direct. In [8] a broadband harmonic sampler is used. The main drawback of this method is the dynamic range since all the noise in the measurement bandwidth of the instrument is integrated into the sampler bandwidth. The spectral filtering approach is proposed in [9-11]. In this method each spectral component to be measured is down-converted to a proper IF where the measurement is easier to perform. An extra channel is dedicated to the acquisition of a reference signal generated in a nonlinear reference. This reference is used to create a grid of frequencies that serves as the phase reference for the spectral regrowth tones. The grid generation has two associated issues. The first one is that all the spectral components involved in the measurement must be correlated with a common reference, forcing the excitation signal to be periodic. The second addresses the fact that each spectral component in the grid must have comfortable energy level. This is difficult to implement since the frequency span needed to capture all the spectral regrowth, both at baseband and at multiple frequencies (considering practical systems at least until fifth order), grows very quickly with the fundamental center frequency.

In this paper a setup intended to measure the input-output characteristics of a nonlinear system presenting a wide bandwidth is proposed. The phase reference is implemented by software giving it a meaningful sense considering the model under extraction. Moreover, it is neither limited by the bandwidth of the input signal nor by the system's operating frequency. In fact, from the system identification point of view, such a procedure can be seen as the systematic extraction of the coefficients of an assumed model.

In the next section, a setup intended to characterize the input-output relation based on an assumed model is presented. Section III shows the simulation validation of the setup and the discussion of the results. Finally, in the Section IV, the conclusions of the work developed are presented.

\section{SETUP ARCHITECTURE}

The setup now presented is intended to overcome the practical difficulty of extracting the nonlinear transfer functions of RF and microwave systems, when a wide channel bandwidth is needed. Moreover, the phase reference can now be obtained by a software operation which overcomes any bandwidth constraints. Since in [12] it was proved that the higher order statistics, $H O S$, can be a useful tool for this model extraction, we are going to find a way to extract the HOS.

In order to calculate the $H O S$, both the input and output signals must be synchronously acquired [12]. However, if a 
wideband signal is considered, it is impossible to acquire an envelope window of the output even with a harmonic sampler such as a microwave transition analyzer. This is due to the fact that the output is composed of, not only the first zone output components, but also the distortion located at multiples of the fundamental frequency. This requires a sampling signal that has an harmonic content up to, at least, twice the signal's highest harmonic involved, and a window length of one period of the envelope. These two conditions lead to a number of points that exceeds the hardware capabilities nowadays available.

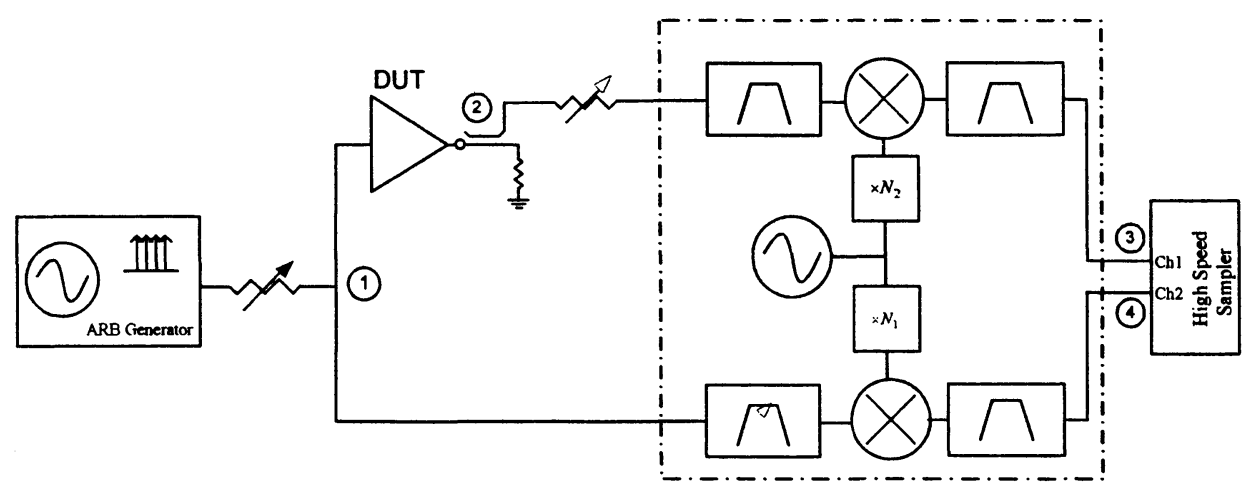

Fig 1. Setup Architecture

The setup now proposed implements a band-selective down-conversion The input signal is down-converted to a proper IF where the non-idealities of the instrumentation produce less impact. This signal can be now acquired and used to synthesize the phase reference. The output of the DUT is also down-converted and acquired. However, it could not be acquired at once, since it spans from several frequency bands depending on the nonlinearity order.

So, each band can now be down-converted according to the frequency multiplication constants $N_{1}$ and $N_{2}$ that should be chosen in order to translate the desired band to the pass band of the IF filter.

The theoretical explanation of this concept starts by assuming the input signal is a multi-sine described by:

$$
x_{i}(t)=\frac{1}{2} \sum_{q=-Q}^{Q} A_{q} e^{j\left(\omega_{q} t+\theta_{q}\right)}
$$

where, $\omega_{q}=\omega_{0}+q \Delta \omega$, with $q \neq 0$

Under the dynamic polynomial or Volterra series models, the signal at the output of the DUT can be given by:

$$
x_{2}(t)=\sum_{n=1}^{N}\left[\frac{1}{2^{n}} \sum_{q_{1}=-Q}^{Q} \ldots \sum_{q_{n=-Q}}^{Q} H_{n}\left(\omega_{q 1}, \ldots, \omega_{q n}\right) A_{q 1} \ldots A_{q n} e^{j\left(\left(\omega_{q 1}+\ldots+\omega_{q n}\right)+\theta_{q 1}(t)+\ldots+\theta_{q n}(t)\right]}\right]
$$

After the down-conversion and filtering, the obtained signal is:

$$
x_{3}(t)=k_{m i x} k_{f i l t}\left[\sum_{n=1}^{N}\left[\frac{1}{2^{n}} \sum_{q_{1}=-Q}^{Q} \ldots \sum_{q_{n=-Q}}^{Q} H_{n}\left(\omega_{q 1}, \ldots, \omega_{q n}\right) A_{q 1} \ldots A_{q n} e^{j\left(\left(\omega_{q 1}+\ldots+\omega_{q n}\right) t+\theta_{q 1}(t)+\ldots+\theta_{q n}(t)\right]}\right]\right] \times \cos \left(N_{2} \omega_{r e f} t+\theta_{r e f}\right)
$$

where: $\omega_{q}^{\prime}=\left(\omega_{0}-N_{2} \omega_{r e f}\right)+q \Delta \omega$, is chosen to be located in the pass-band of the filter by a careful selection of the constant $N_{2}$. The signal in the lower branch of the setup is given by:

$$
x_{4}(t)=k_{\text {mix }} k_{\text {filt }}\left[\frac{1}{2} \sum_{q=-\underline{O}}^{Q} A_{q} e^{j\left(\omega_{q} t+\theta_{q}\right)}\right] \times \cos \left(N_{1} \omega_{\text {ref }} t+\theta_{\text {ref }}\right)
$$

In the same way, $\omega_{q}^{\prime}=\left(\omega_{0}-N_{1} \omega_{r e f}\right)+q \Delta \omega$, is chosen to be in the pass-band of the IF filter.

Adjusting the ratio $N_{2} / N_{1}$ via the change of $N_{2}$, it is possible to select the output spectral band to be down-converted. In order to maintain the spectral coherence between the input and output spectra, the $N_{2} / N_{1}$ relation should be a rational value. Both channels are now converted to the digital domain by a broadband synchronous sampler. Under the digital domain the $H O S$ can now be computed using to the following equation: 


$$
\bar{H}_{n}\left(\omega_{1}, \ldots, \omega_{n}\right)=\frac{S_{y x . x}\left(\omega_{1}, \ldots, \omega_{n}\right)}{S_{x . . x}\left(\omega_{1}, \ldots, \omega_{n}\right)}=\frac{\left\langle Y\left(\omega_{1}+\ldots+\omega_{n}\right)^{*} X\left(\omega_{1}\right) \ldots X\left(\omega_{n}\right)\right\rangle}{\left\langle X\left(\omega_{1}\right) \ldots X\left(\omega_{n}\right) X\left(\omega_{1}\right)^{*} \ldots X\left(\omega_{n}\right)^{*}\right\rangle}
$$

This statistical estimator of the $H_{n}\left(\omega_{1}, \ldots, \omega_{n}\right)$, the $n^{\prime}$ th order Nonlinear Transfer Function, NLTF, assumes that the averages are made through the acquisition of $R$ records of the output and the input when this is a random multi-sine.

\section{SETUP VALIDATION}

In order to validate the proposed idea, a simulation was run in ADS according to Fig. 2. The device under test is a power device transistor that is known to present long-term memory effects. The operating frequency is $900 \mathrm{MHz}$ and the downconversion relation between the two branches, $N_{2} / N_{1}$, is one. The filter was designed to present a $50 \mathrm{MHz}$ bandwidth centered at $40 \mathrm{MHz}$. These specifications are imposed by the used sampler IF bandwidth. The considered input was a three-tone signal, though its generalization to any periodic signal is immediate.

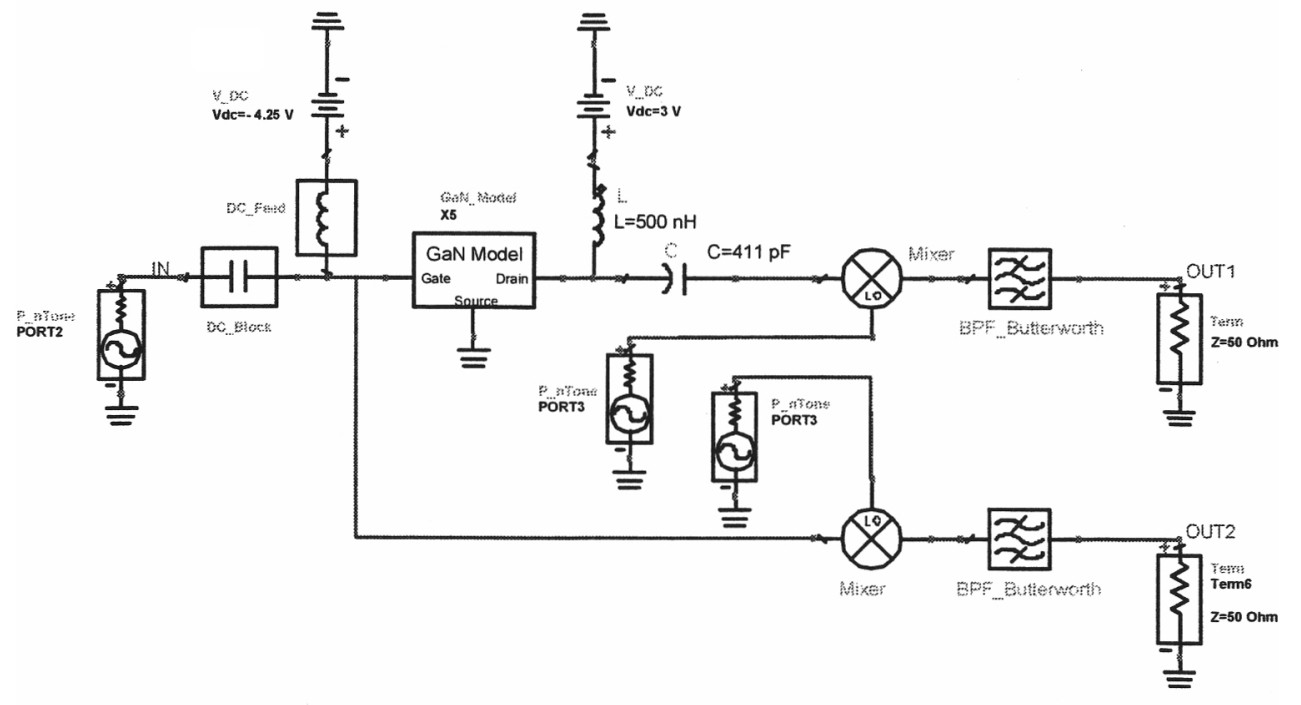

Fig 2. Setup implementation in Ads

In order to calibrate the setup, the DUT is first replaced by a standard through, while the excitation is the desired multisine. So, the calibration consists in measuring the NLTFs of this ideally linear, and of zero delay-length DUT, for the same signal, and for the same amplitude, as observed in the output of the desired DUT.

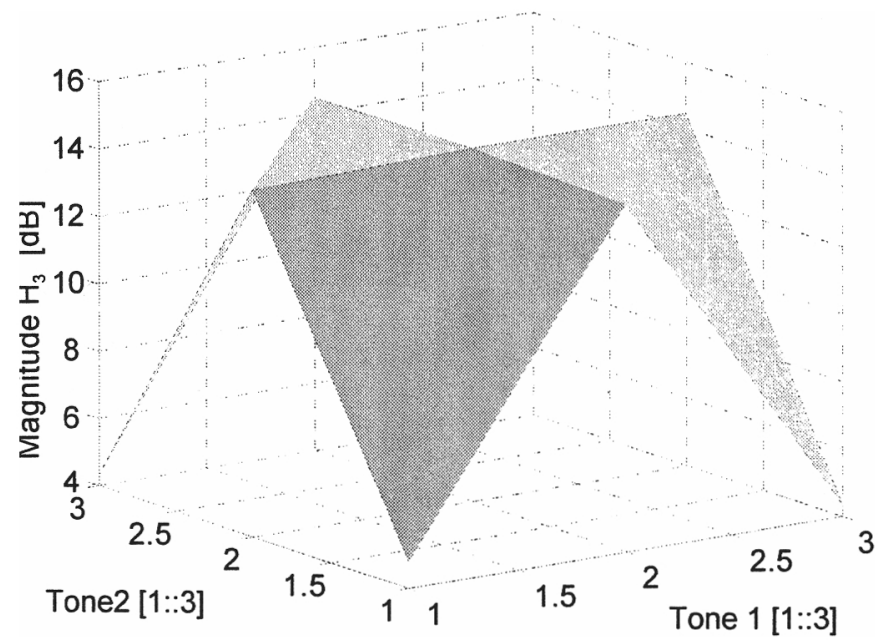

Fig 3. Third order nonlinear transfer function of a $900 \mathrm{MHz}$ power amplifier DUT measured through the setup of Fig. 2. 
Fig. 3 presents the magnitude of $H_{3}\left(\omega_{1}, \omega_{2}, \omega_{3}\right)$ considering a 3 tone signal input for tone $3=-\omega_{2}$. As the result matrix presents three dimensions it is necessary to do a cut in the matrix (tone $3=-\omega_{2}$ ) in order to display the result.

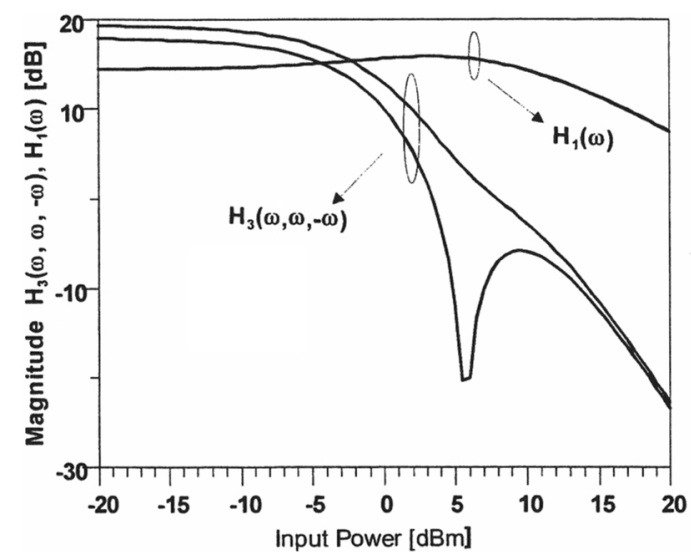

Fig 4. Magnitude of the first and third order transfer functions

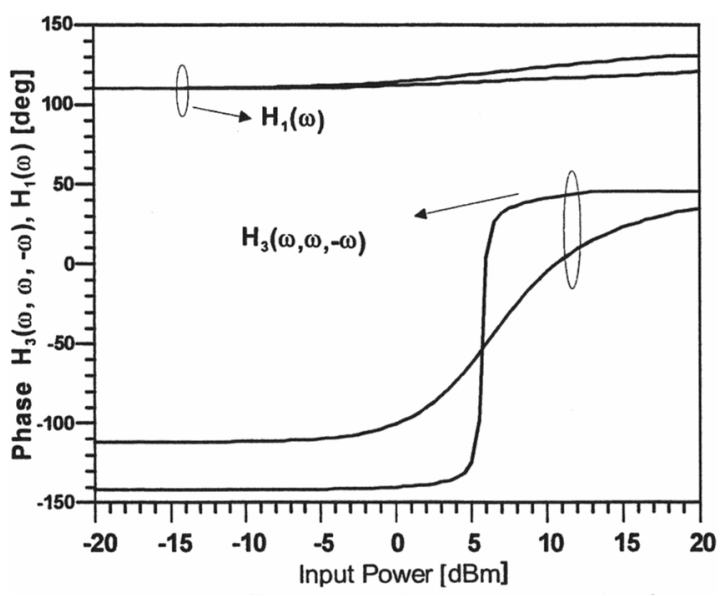

Fig 5. Phase of the first and third order transfer functions

Fig. 4 and Fig. 5 present the magnitude and phase of the first and third order NLTFs, respectively. The evident asymmetry between the upper and lower IMD components is a clear indication of long-term dynamics.

\section{CONCLUSIONS}

The setup now presented permits the nonlinear characterization of a nonlinear circuit or device, circumventing the bandwidth constraints and phase reference limitations encountered in previously proposed instruments. The noise floor of the setup was also improved since the measurement bandwidth can now be controlled by the IF filter's bandwidth. Finally, it should be added that this setup is based on a theoretically supported methodology giving this way a meaningful interpretation, within a nonlinear dynamic model, of the magnitude and phase relations between the output and the input of a nonlinear microwave circuit or device, excited with a multi-sine.

\section{REFERENCES}

[1] K. Remley, D. Williams, D. Schreurs, G. Loglio and A. Cidronali, "Phase Detrending for Measured Multisine Signals", 61st ARFTG Conf. Dig., pp.??-??, Philadelphia, PA, Jun. 2003.

[2] G. Loglio, J. Jargon and D. DeGroot, "Phasor Angle Definition Suitable for Intermodulation Measurements", 65th ARFTG Conference Dig., Jun. 2005.

[3] N. Suematsu, Y. Iyama and O. Ishida, "Transfer Characteristics of IM3 Relative Phase for a GaAs FET Amplifier", IEEE Trans. on Microwave Theory and Tech., Vol. MTT-45, pp.2509-2514, Dec. 1997.

[4] Y. Tang, J. Yi, J. Nam, B. Kim and M. Park, "Measurement of Two-Tone Transfer Characteristics of High-Power Amplifiers", IEEE Trans. on Microwave Theory and Tech., Vol. MTT-49, pp.568-571, Mar. 2001.

[5] J. Vuolevi, T. Rahkonen and J. Manninen, "Measurement Technique for Characterizing Memory Effects in RF Power Amplifiers" IEEE Trans. on Microwave Theory and Tech., Vol. MTT-49, pp.1383-1389, Aug. 2001.

[6] J. Vuolevi and T. Rahkonen, Distortion in RF Power Amplifiers, Artech House, Norwood, MA, 2003.

[7] J. Dunsmore and D. Goldberg, "Novel Two-Tone Intermodulation Phase Measurement for Evaluating Amplifier Memory Effects," 33rd European Microwave Conf., Oct. 2003, pp. 235-238.

[8] J. Verspecht, F. Verbeyst and M. Vanden Bossche, "Network Analysis Beyond S-Parameters: Characterization and Modelling Component Behaviour under Modulated Large-Signal Operating Conditions", $56^{\text {th }}$ ARFTG Conference Proceedings, Colorado (USA), December 2000

[9] J. Martins and N. Carvalho, "Spectral Filtering Setup for Uncorrelated Multi-tone Phase and Amplitude Measurement", 34'th European Microwave Conf. Proc., pp.201-204, Amsterdam, Oct. 2004.

[10] J. Martins and N. Carvalho, "Multitone Phase and Amplitude Measurement for Nonlinear Device Characterization", IEEE Trans. on Microwave Theory and Tech., Vol. MTT-53, pp.1982-1989, Jun. 2005.

[11] Blockley, P.; Gunyan, D.; Scott, J.B.,'Mixer-based, Vector-Corrected, Vector Signal/Network Analyzer Offering 300kHz-20 GHz Bandwidth and Traceable Phase Response" ", IEEE Intern. Microwave Symp. Dig., pp.1497-1500, Long Beach, Jun. 2005.

[12] J. Pedro, J. Martins and P. M Cabral, "New Method for Phase Characterization of Nonlinear Distortion Products", IEEE Intern. Microwave Symp. Dig., pp.971-974, Long Beach, Jun. 2005. 